\title{
Studying the Interrelation of the Physical and Mechanical Properties of Artificial Grass Composites Affecting its Performance
}

\author{
Magdi El-Messiry ${ }^{1}$, Samar Abdelatif ${ }^{2}$, Rania El-Deeb $^{3}$ and Sherine El- \\ Tarahony $^{4 *}$
}

Textile Engineering Department, Faculty of Engineering, Alexandria University, Alexandria, 21544, Egypt

*Sherine El-Tarahony

Sherine.eltarahony@gmail.com

\begin{abstract}
In this paper, the advantages of using the artificial grass composites instead of the natural grass are going to be discussed. FIFA sets down the rules of the game which cover the ball's weight, material, circumference, sphericity, pressure and also, the use of the artificial grass composites. Artificial grass composites were introduced as an alternative to natural turf in an attempt to overcome some issues such as shortage of water, increased participation in sports and high operational and maintenance costs. Hence, it is important to know the regulations and the quality controls of the ball that can be used in the game. This can be done by developing a theoretical approach describing the behavior of the artificial grass, developing the parameters recommendation catalog and designing the football end-use artificial grass according to FIFA regulations.

In this work, the physical and mechanical properties of various artificial grass carpets with different pile heights, raw materials, thickness, pile count, and density are investigated. A tester to measure the vertical ball rebound height, oblique ball rebound height and the coefficient of restitution (COR) are introduced. In addition, a tester to measure the residual impact force (RIF) of the ball is proposed. Taking into consideration the reaction of the standard football on the surface of the artificial grass composites (Ball / Surface interaction) with the different specifications of the grass, a theoretical model describing the artificial grass behavior as a function of the artificial grass different parameters is developed. This model can be used to design the artificial grass combination parameters that are compliant with FIFA recommendation regulations (FIFA $1^{*}$ and FIFA $2 *$ ). In addition, further improvement can be done by studying more parameters that affect the artificial grass design and behavior such as ball roll, joint strength, underfoot friction, abrasion resistance, and grass permeability. A similar line of work can be applied to different end-uses such as landscaping, golf, and rugby.
\end{abstract}

Keywords: Artificial grass, Ball rebound height, Coefficient of restitution (COR), Residual impact force (RIF), FIFA $1 * / 2 *$ 


\section{Introduction}

Artificial turf surfaces became recognised in 1965 when AstroTurf TM was installed at the Astrodome in Houston, Texas. The use of this artificial sports surface spread widely in the 1980's and was installed in both outdoor and indoor stadiums for football and baseball in North America. The acceptance of artificial turf surface varies with different sports. The transition from natural to artificial turf surface has completed for the game of field hockey as the majority of top level competitions are played on artificial turf [1].In other sports such as soccer and rugby, this transition has been much slower. However, since the 1990's, the newer (third) generation of artificial turf offers playing characteristics closer to natural turf and has permitted its use for professional and international matches sanctioned by international governing bodies[2].

The 'Quality Concept' has been established since 1st January 1996 and is part of the Denominations Programme initiated by FIFA in 1995 [3] [4].The idea was to introduce a high quality and consistent ball which would be distinguished through one of the Quality Marks included in the ball graphics. The laboratory-based test procedure is mainly concerned with the static based testing of footballs. Additionally, FIFA has now also developed a Quality Concept for the use of artificial turf surfaces as an alternative to the usual natural turf used for games[5].The artificial pitches are designed according to the desired application either specifically for individual sports or as multi-sports surfaces. Artificial turf system is an installation composed of several layers, and each layer is made from different materials. However, all the outdoor pitch systems share a basic structure. The system can be split into three major groups: foundation layer, shock-pad layer and carpet layer. They are distinguished by their functions in the pitch performance and materials used for their construction [6].

The quality of the raw materials is crucial to the performance of turf systems. Almost anything used as a carpet backing has been used for the backing material, from jute to plastic to polyester. High quality artificial turf uses polyester tire cord for the backing. The fibers that make up the blades of "grass" are made of nylon or polypropylene and can be manufactured in different ways. The nylon blades can be produced in thin sheets that are cut into strips or extruded through molds to produce fibers with a round or oval cross-section. The extruded product results in blades that feels and act more like biological grass. Cushioning systems are made from rubber compounds or from polyester foam. Rubber tires are sometimes used in the composition of the rubber base, and some of the materials used in backing can come from plastic or rubber recycling programs. The thread used to sew the pads together and also the top fabric panels has to meet the same criteria of strength, color retention, and durability as the rest of the system. Care and experience must also be applied to the selection of the adhesives used to bond all the components together [7].

The "grass" part of a turf system is made with the same tufting techniques used in the manufacture of carpets. The first step is to blend the proprietary ingredients together in a hopper. Dyes and chemicals are added to give the turf its traditional green color and to protect it from the ultraviolet rays from the sun then extrusion, after extrusion, the yarns are either twisted on their own axis to become a rounder and stronger shape, or one yarn is wrapped around a thinner yarn to give the strengthening. These finished yarns are wrapped around spools and fed into the tufting machine. The tufting process utilizes a large needle which pushes through the backing material to create a loop that is later cut to create the final pile then installation and finishing then safety[7]. 
As mentioned in the abstract above it's important to know the regulations and the quality controls concerning the ball to be used in the game by developing a theoretical approach describing the behavior of the artificial grass, developing the parameters recommendation catalog and designing the football end-use artificial grass according to FIFA regulations (FIFA $1 *$ or FIFA $2 *$ ).

\section{Materials and Methods}

Laboratory tests shall be made at an ambient laboratory temperature of $23 \pm 2^{\circ} \mathrm{C}$. Test specimens shall be conditioned for a minimum of 3 hours at the laboratory temperature prior to test [8].19 types of samples different in pile height, density, colour, material, origin, count, gauge and end-use were used. The samples have been imported from China, the UK and Egypt as shown in Table 1. Some of these samples can be used in Sports like Football, Hockey or Rugby, etc. And some of them can be used for Landscaping, some of the materials are made of polypropylene, polyethylene or mixed of polypropylene and polyethylene. Two types of rubbers with thickness $2 \mathrm{~mm}$ and $4 \mathrm{~mm}$ were used and tested under the original sample which has $0.5 \mathrm{~mm}$ rubber[9][10][11].

Table 1: Artificial grass composites samples specifications

\begin{tabular}{|l|l|l|l|l|l|l|}
\hline & $\begin{array}{l}\text { Samples } \\
\text { Name }\end{array}$ & Material & $\begin{array}{l}\text { Pile } \\
\text { Height } \\
\text { (cm) }\end{array}$ & $\begin{array}{l}\text { Densit } \\
\mathbf{y}(\mathbf{s t i t c} \\
\text { hes/sq } \\
\mathbf{c m})\end{array}$ & Gauge(cm) & $\begin{array}{l}\text { Count } \\
\text { (Tex) }\end{array}$ \\
\hline $\mathbf{1}$ & SE01X & PE+PP & 2.5 & 13.65 & 0.9525 & 13 \\
\hline $\mathbf{2}$ & SE02X & PE & 2.5 & 13.65 & 0.9525 & 12.5 \\
\hline $\mathbf{3}$ & SE03X & PE+PP & 2.7 & 14 & 0.9 & 13 \\
\hline $\mathbf{4}$ & SE04X & PE+PP & 3 & 14.5 & 0.95 & 13 \\
\hline $\mathbf{5}$ & SE05X & PE & 3 & 16.8 & 0.9525 & 11 \\
\hline $\mathbf{6}$ & SE06X & PE & 3 & 16.8 & 0.9525 & 11.5 \\
\hline $\mathbf{7}$ & SE07X & PE+PP & 3 & 13.65 & 0.9525 & 11.5 \\
\hline $\mathbf{8}$ & SE08X & PE+PP & 3 & 16.8 & 0.9525 & 9.2 \\
\hline $\mathbf{9}$ & SE09X & PE+PP & 3.4 & 14.5 & 0.8 & 13 \\
\hline $\mathbf{1 0}$ & SE10X & PE+PP & 3.5 & 15 & 0.95 & 12.5 \\
\hline
\end{tabular}




\begin{tabular}{|l|l|l|l|l|l|l|}
\hline $\mathbf{1 1}$ & SE11X & PE & 3.5 & 18.9 & 0.9525 & 13 \\
\hline $\mathbf{1 2}$ & SE12X & PE+PP & 4 & 15.75 & 0.9525 & 11.5 \\
\hline $\mathbf{1 3}$ & SE13X & PE & 5 & 9.45 & 0.9525 & 11 \\
\hline $\mathbf{1 4}$ & SE14X & PE & 5 & 10.08 & 0.9525 & 8.8 \\
\hline $\mathbf{1 5}$ & SE15X & PE & 5 & 10.71 & 1.5875 & 11 \\
\hline $\mathbf{1 6}$ & SE16X & PE & 5 & 9.45 & 1.5875 & 11 \\
\hline $\mathbf{1 7}$ & SE17X & PE & 5 & 9.45 & 1.5875 & 12.5 \\
\hline $\mathbf{1 8}$ & SE18X & PE & 5 & 8.82 & 1.5875 & 11 \\
\hline $\mathbf{1 9}$ & SE19X & PP & 5.3 & 9.45 & 1.8 & 11 \\
\hline
\end{tabular}

\section{Experimental Work}

\subsection{Measuring residual ball surface impact force and ball rebound height of artificial grass.}

A tester is introduced to measure the vertical and oblique ball rebound height by capturing the images of the experiment and recording the videos. the ball was hanged by a clamp on a stand $2.0 \mathrm{~m}$ high and once the ball was freed, the video started to record the ball while dropping on the artificial grass sample. Ball rebound height shall be measured using the test procedure specified in EN 12235. In this test the ball is released without imparting any impulse or spin from a height of $2.0 \mathrm{~m}$ and the rebound from the bottom of the ball to the surface was measured. Every material includes rubber base, the thickness of this rubber base was increased by $2 \mathrm{~mm}$ and $4 \mathrm{~mm}$. Measuring the oblique ball rebound height, tests were done with ball inclined by 70 degrees with the artificial grass on the ground and ball inclined by 50 degrees with the artificial grass on the ground. Every material has its own rubber base with $0.5 \mathrm{~mm}$ thickness, by adding $2 \mathrm{~mm}$ rubber and $4 \mathrm{~mm}$ rubber separately on each sample the ball rebound height were measured while the ball is inclined by 70 degrees with the artificial grass on the ground and inclined by 50 degrees with the artificial grass on the ground. In order to measure the residual impact force, a testing setup was designed as shown in figure 1 (b), the ball was dropped vertically from $2.0 \mathrm{~m}$ height on different types of materials, 3 times per one sample and with very high-speed imaging and video capturing, the data was analyzed to get the residual impact force value when the ball hit the grass. This test was done using the normal rubber under the grass material which was $0.5 \mathrm{~mm}$ then by adding extra rubber base of $2 \mathrm{~mm}$ and $4 \mathrm{~mm}$ to show the effect of thickness on residual impact force putting in to consideration some energy absorption and end-use of each material whether it is football enduse or any other end-use. Some specific parameters needed to be calculated after measuring the ball rebound height and residual impact force such as coefficient of restitution, potential 
energy into kinetic energy of dropped ball conversion, modulus of elasticity, buckling force and rubber properties.

Figure 1 - (a) Ball Rebound Height Tester; (b) Residual Impact Force Tester
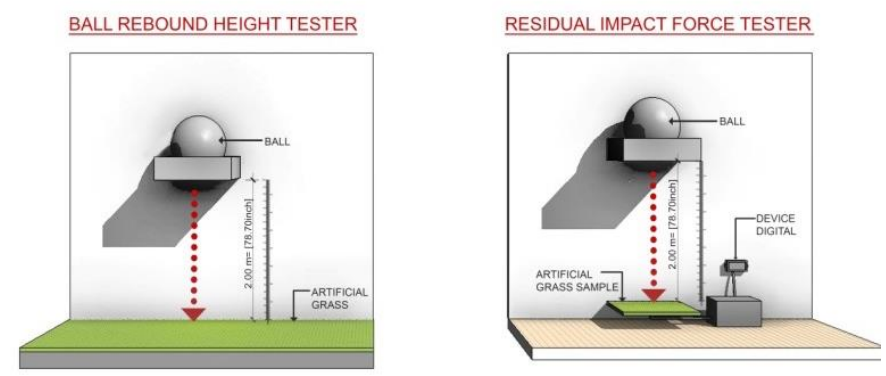

\section{Results and discussion}

\subsection{Vertical / Oblique ball rebound height and coefficient of restitution (COR)}

In this paper two testers were designed to measure vertical / oblique ball rebound height and residual impact force of artificial grass composite in football end-use as shown in Figure 1 (a) and Figure 1 (b).

\section{Figure 2 - Vertical ball rebound height for different rubber thicknesses}

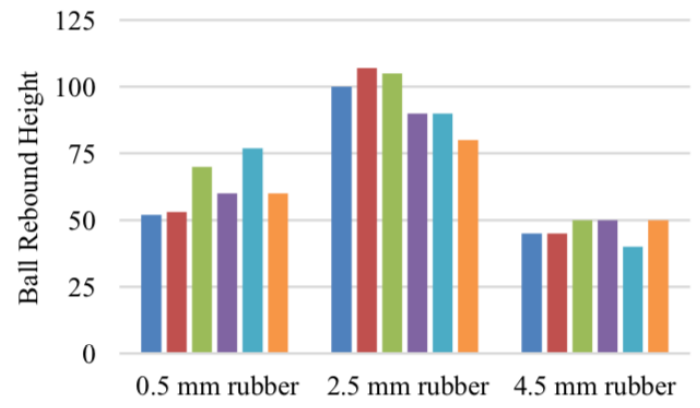

Figure 3 - Oblique ball rebound height at angle 50 degrees and different rubber thicknesses

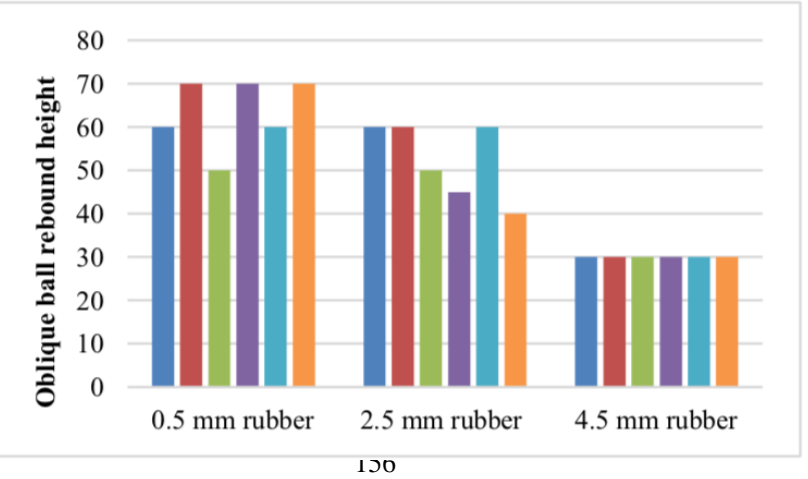




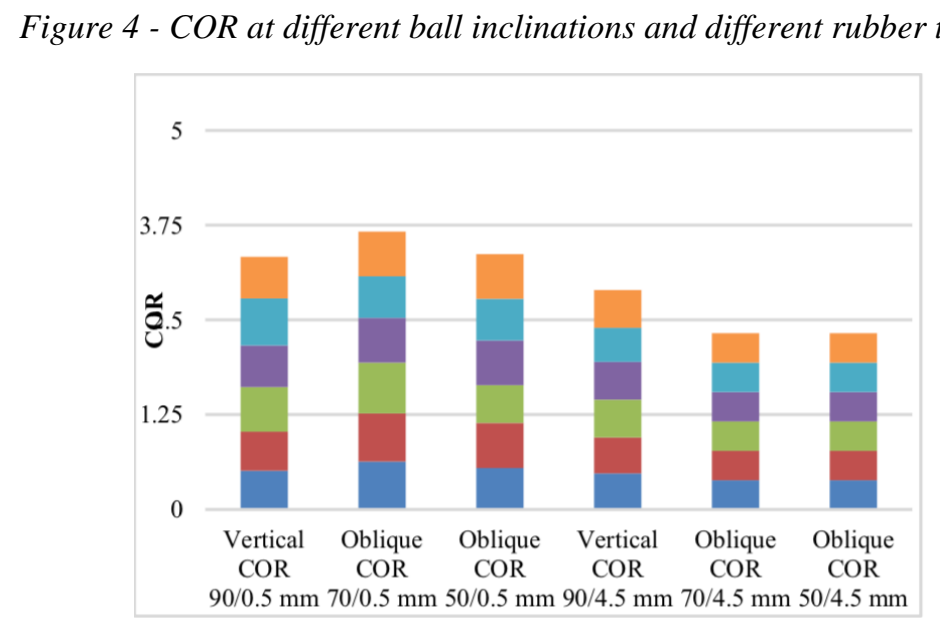

In the football end-use, Figure $\mathbf{2} \mathbf{\& 3}$ showed that the ball rebound height increased when adding more rubber till it comes to the peak point at $2.5 \mathrm{~mm}$ rubber thickness then start to decrease gradually till it becomes constant. From the ONE-WAY ANOVA test, the results showed that the two factors are significantly effective. While the oblique ball rebound height at ball inclination 70 and 50 degrees, the ball rebound height decreases with the increase of rubber thickness base till it becomes constant. From the two-way ANOVA without replication test, the results showed that the three factors are significantly effective.

Finally, It was proved that the coefficient of restitution (COR) is an important parameter to be calculated and it showed the relation between rebound height and initial height. Figure 4 gathered the COR value at different cases of ball inclination and rubber thicknesses. $0.5 \mathrm{~mm}$ rubber thickness is within the FIFA $1^{*}$ range and $4.5 \mathrm{~mm}$ rubber are within the FIFA $2 *$ range.

\subsection{Factors affecting the residual impact force (RIF)}

In the football End-Use, for the case of $0.5 \mathrm{~mm}$ rubber base results showed that the residual impact force is directly proportional with count(Tex) with $\mathrm{R}^{2}$ value of 0.7761 and linear regression equation.

$$
\mathrm{y}=2.5529 \mathrm{x}-13.153
$$

While for the case of $4.5 \mathrm{~mm}$ rubber base. $\mathrm{R}^{2}$ value 0.985 and linear regression equation.

$$
\mathrm{y}=2.7334 \mathrm{x}-2.3462
$$

In the football End-Use, for the case of $0.5 \mathrm{~mm}$ rubber base results showed that the residual impact force is inversely proportional with Density with $\mathrm{R}^{2}$ value of 0.9898 and linear regression equation.

$$
y=-10.658 x+120.04
$$

While, for the case of $4.5 \mathrm{~mm}$ rubber base $\mathrm{R}^{2}$ value 0.6628 and linear regression equation.

$$
y=-3.373 x+44.5
$$


In the football End-Use, for the case of $0.5 \mathrm{~mm}$ rubber base results showed that the residual impact force is directly proportional with modulus of elasticity (E) with $R^{2}$ value of 0.6205 and linear regression equation.

$$
\mathrm{y}=19.146 \mathrm{x}-10.941
$$

While, for the case of $4.5 \mathrm{~mm}$ rubber base $\mathrm{R} 2$ value 0.9215 and linear regression equation.

$$
\mathrm{y}=48.237 \mathrm{x}-56.812
$$

In the football End-Use, for the case of $0.5 \mathrm{~mm}$ rubber base, results showed that the residual impact force is inversely proportional with buckling force with value $\mathrm{R}^{2}=0.9165$ and linear regression equation.

$$
\mathrm{y}=-279.68 \mathrm{x}+55.849
$$

While, for the case of $4.5 \mathrm{~mm}$ rubber base $\mathrm{R}^{2}$ value 0.9888 and linear regression equation.

$$
y=-29.681+21.681
$$

\subsection{Analysis of residual impact force measured, and calculated parameters results at football End-Use (Empirical formula)}

$$
\text { Residual Force }=\mathrm{K}\left(\frac{\text { Buckling Force } * \text { Density }}{\text { Young'sModulus } * \text { Tex }}\right)^{-5.96}
$$

Where $\mathrm{K}=1.1 * 10^{-6}($ thickness of the rubber+ 5 )

\section{Figure - 5 - Relation between measured and calculated RIF}

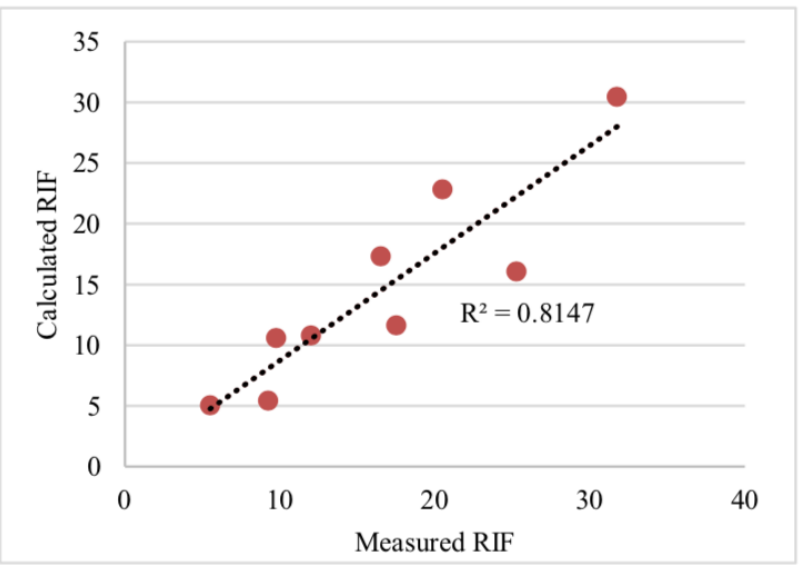

Figure 5 showed the relation between the measured RIF from the designed tester and calculated RIF from the empirical formula with $\mathrm{R}^{2}=0.8147$ at $0.5 / 4.5 \mathrm{~mm}$ rubber base based on FIFA standards. 


\subsection{Relation between residual impact force (RIF) and coefficient of restitution (COR) at Football End-Use according to the FIFA standards.}

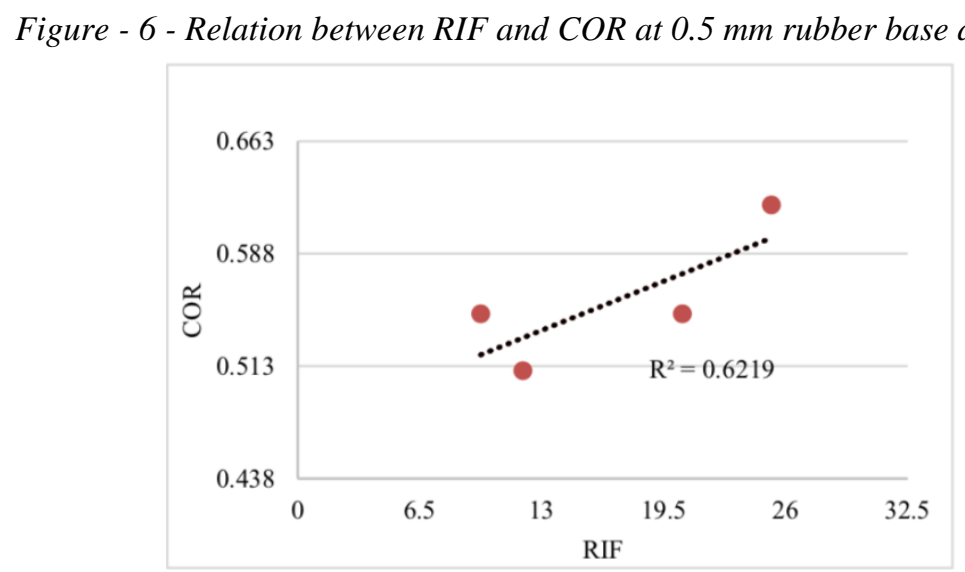

Fig.7 - Relation between RIF and COR at $4.5 \mathrm{~mm}$ rubber base and FIFA $2 *$

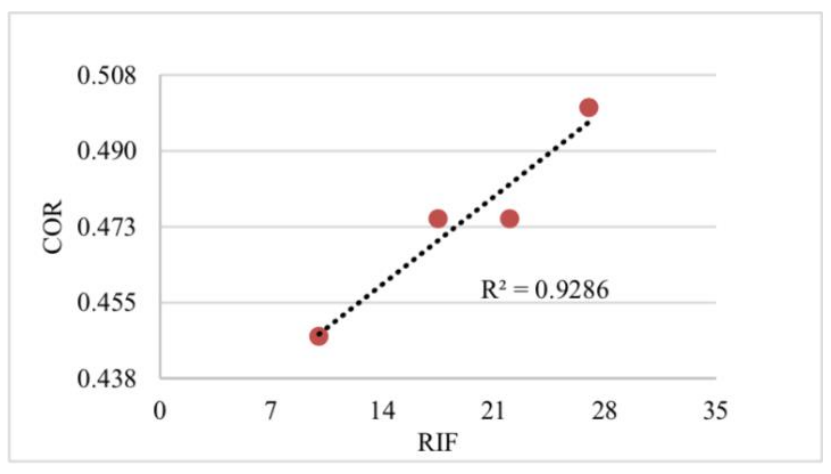

In Figure 6 showed directly proportional linear equation between RIF and COR at first case of $0.5 \mathrm{~mm}$ rubber base with $\mathrm{R}^{2}=0.6219$ and first-degree equation.

$$
\mathrm{y}=0.005 \mathrm{x}+0.0 .4716
$$

While, in Figure 7 at second case of $4.5 \mathrm{~mm}$ rubber base showed directly proportional linear equation between RIF and COR with $\mathrm{R}^{2}=0.9286$ and first-degree equation.

$$
\mathrm{y}=0.0029 \mathrm{x}+0.4189
$$

which will achieve the FIFA $1 *$ and $2 *$ requirements while in case of $2.5 \mathrm{~mm}$ rubber base, it didn't achieve the FIFA requirements and showed results out of the FIFA range. 


\section{Conclusions}

Mechanical and physical parameters such as Ball Rebound Height and thus, Coefficient of Restitution, Residual Impact Force, Pile Height, Grass Density, Yarn Count, Modulus of Elasticity, Yarn Buckling Force were tested to show the effect of the artificial grass types on its behaviour. Yarn count, modulus of elasticity were found to be directly proportional to the residual impact force acting on the football. Grass density and yarn buckling force were found to be inversely proportional to the residual impact force acting on the football. Grass density and yarn buckling force were found to be inversely proportional to the residual impact force acting on the football. Using extra $2 \mathrm{~mm}$ and $4 \mathrm{~mm}$ rubber thicknesses under the original rubber thickness of $0.5 \mathrm{~mm}$ showed an impressive effect while measuring the vertical and oblique ball rebound heights. An empirical equation relating RIF and the artificial grass specifications was worked out and the calculated values were found to be significantly representing the measured value with an $\mathrm{R}^{2}$ of 0.817 at $0.5 / 4.5$ rubber thicknesses. The ball rebound height has also been tested to show its direct proportional relationship with the residual impact force and accordingly, with the coefficient of restitution based on the FIFA Quality marks. A case study was performed according to the empirical equation from which a catalogue for the most important parameters representing the artificial grass was prepared. By adding more rubber $(2 \mathrm{~mm}$ or $4 \mathrm{~mm}$ thickness) under the artificial grass sample which has already $0.5 \mathrm{~mm}$ rubber base. The $0.5 \mathrm{~mm}$ rubber base achieved the FIFA $1 *$ standards, the 4.5 mm rubber base achieved the FIFA $2 *$ standards while the $2.5 \mathrm{~mm}$ rubber base didn't achieve the regulations and showed results out of the range.

\section{Acknowledgments}

The authors of this paper acknowledge the support of Viva Turf (China), Nam grass (UK) and oriental weavers (Egypt) in supplying the artificial grass composites materials. 


\section{References}

[1] FIH, "Handbook of Performance Requirements for Synthetic Hockey Pitches: The Pitch Handbook. Lausanne, Switzerland.," 2008.

[2] A. D. T. in L. U. Wang, Xinyi, "Advanced measurement for sports surface system behaviour under mechanical and player loading," 2013.

[3] FIFA, "FIFA (Ball manufacture, History and BigCount Survey)," 2007. [Online]. Available: www.FIFA.com.

[4] FIFA, "FIFA Quality Concept - artificial turf football surfaces; February 2005," 2005. [Online]. Available: www.fifa.com.

[5] D. S. Price, "Advanced Modelling of Soccer Balls, Doctor of Philosophy, Loughborough University.," Loughborough University, 2005.

[6] L. Anderson, "Elastomeric Shockpads for Outdoor Synthetic Sports Pitches, PhD Thesis.," Loughborough University,UK., 2007.

[7] How products are made, "Artificial Turf." [Online]. Available: http://www.madehow.com/Volume-7/Artificial-Turf.html.

[8] FIH, "Handbook of Performance, Durability and Construction Requirements for Synthetic Turf Hockey Pitches," Lausanne,Switzerland, 2014.

[9] "VIVA TURF,CHINA," 2015. [Online]. Available: http://www.vivaturf.com/.

[10] “ORIENTAL WEAVERS,EGYPT," 2016. [Online]. Available: http://www.orientalweavers.com/.

[11] “NAM GRASS,UK,” 2016. [Online]. Available: https://www.namgrass.co.uk/. 